\title{
L'argumentation dans la formation des groupes protestataires : du conflit d'aménagement au militantisme environnemental
}

Argumentation in the Formation of Protest Groups: from Land-Development Conflict to Environmental Activism

\section{Aurélien Allouche}

\section{(2) OpenEdition Journals}

Electronic version

URL: http://journals.openedition.org/aad/1929

DOI: $10.4000 /$ aad. 1929

ISSN: 1565-8961

Publisher

Université de Tel-Aviv

\section{Electronic reference}

Aurélien Allouche, «L'argumentation dans la formation des groupes protestataires : du conflit

d'aménagement au militantisme environnemental », Argumentation et Analyse du Discours [Online], 14 2015, Online since 09 April 2015, connection on 23 September 2019. URL : http://

journals.openedition.org/aad/1929; DOI : 10.4000/aad.1929

This text was automatically generated on 23 September 2019.

\section{cc)}

Argumentation \& analyse du discours est mis à disposition selon les termes de la licence Creative Commons Attribution - Pas d'Utilisation Commerciale - Pas de Modification 4.0 International. 


\title{
L'argumentation dans la formation des groupes protestataires : $d u$ conflit d'aménagement au militantisme environnemental
}

\author{
Argumentation in the Formation of Protest Groups: from Land-Development \\ Conflict to Environmental Activism
}

Aurélien Allouche

\section{AUTHOR'S NOTE}

L'auteur remercie Marianne Doury, chercheure au Laboratoire Communication et Politique (LCP) pour ses conseils précieux aboutissant à cet article

1 Les mouvements environnementalistes se sont en grande partie constitués à la suite de mobilisations contre des projets d'aménagement (Cans 2006). Au départ, ces conflits sont souvent des protestations collectives sans revendication autre que le refus d'une infrastructure par les riverains et usagers du lieu. L'obligation de forger un argumentaire pour justifier ce refus peut faire évoluer ces mobilisations vers le militantisme environnementaliste. La nécessité pour les acteurs protestataires d'élaborer un argumentaire commun prenant en charge l'intérêt général les conduit à définir ce qui peut les unir au-delà de leur refus premier et, éventuellement, à aligner leur position sur le militantisme d'une fraction d'entre eux (Lafaye \& Thevenot 1993; Simon 2010). On peut dès lors questionner le rôle exact que l'argumentation joue dans la transition des mobilisations protestataires vers le militantisme environnementaliste. Nous abordons cette problématique sur le plan théorique en articulant trois lignes d'analyse qui se détachent plus spécifiquement de la littérature portant sur ce sujet, articulation que nous confronterons ensuite à l'étude empirique d'un conflit d'aménagement: le rôle de l'interdiscursivité dans l'évolution des engagements 
militants, la contribution des arguments ad hominem à la constitution d'un ethos militant, enfin, l'investissement des échanges argumentatifs comme une forme de sociabilité et de convergence entre militants.

\section{Cadre théorique}

\subsection{Le rôle de l'interdiscursivité}

2 En premier lieu, l'action collective suppose des acteurs un travail constant d'articulation de répertoires d'arguments aux formes prises par leur action (Cefaï et Lafaye 2001; Stewart, Smith et Denton 2007), de sorte que les formes prises par les mobilisations collectives se dessinent dans la mise en relation complexe du jeu d'acteurs et du jeu d'arguments (Chateauraynaud 2007, 2011). Jeu d'arguments et jeu d'acteurs ne se correspondant pas nécessairement et suivant des logiques propres, un espace-temps particulier s'ouvre ainsi à l'analyse. Pour Juliette Rennes (2011), reprenant les travaux de Mark Steinberg, l'articulation de ces deux niveaux de la réalité sociale repose sur l'interdiscursivité, laquelle est comprise comme l'interdépendance des discours des parties prenantes d'un conflit. Notre optique n'est pas celle d'étudier la constitution complexe des jeux d'acteurs ni les phénomènes d'influence réciproques entre arènes publiques ou entre controverses. C'est en quelque sorte à la face interne de ces processus que nous nous intéressons ici, à leurs résultantes sur les dynamiques internes des groupes. Or outre la circulation d'arguments entre des groupes ou des arènes publiques, l'interdiscursivité, dans le cadre de notre problématique, nous permet de voir comment l'intégration d'arguments adverses par un groupe amène insensiblement celui-ci à faire évoluer la nature de ses revendications, son engagement et la façon dont il comprend l'action qu'il mène. La relecture des travaux de Bernard Groethuysen (1977) par Alban Bouvier $(1997,1999)$ le suggère également, en montrant l'effet, au 19e siècle, de la controverse déclenchée par la condamnation par l'Église du prêt à intérêt sur la formation d'une éthique capitaliste et bourgeoise à mesure que, d'argument en contre-argument, les opposants au clergé viennent à former les principes d'une éthique profane pour justifier la pratique du prêt à intérêt.

3 L'interdiscursivité permet de comprendre que des acteurs, initialement réunis dans le seul but de s'opposer aux désagréments d'un aménagement, en viennent, d'arguments en contre-arguments, à énoncer des principes et des valeurs environnementalistes qu'ils finiront par assumer et par revendiquer, bien au-delà de leur motif premier de protestation, ou encore à rapprocher d'autres causes de la leur.

\subsection{Arguments ad hominem et ethos militant}

4 Cette interdiscursivité facilite un second type de processus. L'argumentation des militants se fonde souvent sur l'ethos, au sens rhétorique du terme, c'est-à-dire au sens de l'image de soi qu'un locuteur s'emploie à construire et à mobiliser pour rallier son auditoire à la thèse qu'il défend (Amossy 2006). Ce « travail de leur ethos » interagit avec la construction de l'identité collective et la dynamique organisationnelle des groupes militants (Orkibi 2008).

5 Dans les conflits d'aménagement l'ethos rhétorique s'aborde en des termes similaires bien que spécifiques. Dans ces conflits, les controverses portent en grande partie sur 
des dimensions techniques et des données factuelles, donc a priori peu propices à la mobilisation de tels éléments rhétoriques. Pourtant, dans ces controverses, des points peuvent demeurer indécidables ou impossibles à argumenter de manière factuelle. Il n'est pas rare alors que les débatteurs recourent à des arguments ad hominem pour convaincre, c'est-à-dire à des arguments réfutant ou défendant une proposition en substituant à la discussion du contenu des énoncés l'évocation des caractéristiques des énonciateurs ou de la situation d'énonciation. L'ethos des débatteurs devient alors central. La technicité des propositions débattues fait toutefois que les vertus que tendront à manifester ces débatteurs sont d'un genre particulier. Les acteurs doivent en effet manifester les qualités personnelles qui assurent à l'interlocuteur qu'ils sont à la fois capables d'accéder à la factualité et de la restituer, et désireux de le faire honnêtement.

6 Analyser le rôle de l'argumentation dans l'émergence du militantisme au cours de conflits d'aménagement revient ici à se demander dans quelle mesure les arguments ad hominem conduisent les militants, à l'occasion de débats qui prennent tous les traits de la discussion technique de l'acceptabilité d'infrastructures, à revendiquer des vertus personnelles dont la justesse de leur jugement peut se prévaloir. Un renforcement de l'attitude militante des acteurs protestataires peut s'ensuivre. La revendication de vertus épistémiques (Zagzebski 2005) et l'affichage des traits supposés les manifester peuvent amener les militants à se percevoir eux-mêmes comme tels et, partant, s'attribuer un statut particulier dans l'espace public. D'autre part, le plus sûr moyen pour un acteur de convaincre son auditoire de vertus particulières est de les adopter effectivement dans sa conduite ordinaire (Amossy 2006 :72). La logique de l'ethos rhétorique communique, par ce biais, avec celle de l'ethos défini cette fois en termes sociologiques comme une "économie globale de la conduite de la vie» (Weber 1963 : 200).

\subsection{L'argumentation comme forme de convergence et de sociabilité entre militants}

7 Enfin, la défense d'un argumentaire commun occupant une place centrale dans l'activité des groupes protestataires, les échanges argumentatifs peuvent être investis par les militants comme un mode de relation sociale. La vie d'un collectif d'opposition est régulièrement marquée par des épisodes d'argumentation collective, visant à étayer la position commune et à réfuter les arguments que ce collectif sait lui être opposés. Les militants se trouvent alors en situation d'argumenter ensemble leurs propres propositions. Les acteurs participant à ces échanges sont a priori déjà acquis à la cause pour laquelle ils se sont engagés et déjà convaincus des propositions pour lesquelles ils militent, tout du moins existe-t-il un ensemble a minima de propositions que chacun d'entre eux s'est engagé à défendre en tant que membre du groupe. Ces échanges alors se détachent de la fonction persuasive de l'argumentation. Or Marianne Doury (2012) a montré que la fonction persuasive n'est pas nécessaire à la définition de l'argumentation. Plus encore, se départir de cette réduction théorique rend analysables différents types d'échanges, tels que ceux consistant à " prêcher des convertis ». Les échanges argumentatifs entre militants déjà convaincus deviennent dès lors pertinents pour l'analyse sociologique. Ces échanges, plutôt que d'être des dérives ratiocinatrices de l'action collective, s'inscrivent plus largement dans l'ensemble des formes discursives routinières que des acteurs, conjointement engagés dans une action 
collective, peuvent investir pour converger vers une intention commune et vers la définition de contenus qu'ils partagent (Conein 2005). De surcroit, ces échanges peuvent être investis comme une forme de sociabilité dont l'attrait central est le plaisir de partager une même conviction avec d'autres personnes.

Il convient de se placer à un niveau empirique pour discuter l'articulation de ces trois lignes d'analyse. On s'appuie pour cela sur le suivi, de 2004 à 2007, d'un collectif de dixneuf associations opposées à la construction d'un canal reliant une centrale hydroélectrique, située au nord de l'étang de Berre (Bouches-du-Rhône) au Rhône, qui s'écoule à une vingtaine de kilomètres à l'ouest de cet étang. Ce suivi a consisté en une observation participante mettant l'accent sur les moments durant lesquels les membres du collectif travaillaient à un argumentaire commun, à l'analyse de différentes données (études hydrauliques, rapport d'expertise, etc.) ou à l'organisation d'événements publics. Le matériau discursif ainsi recueilli est constitué de la retranscription intégrale de trente-quatre réunions de travail dont sont issus les deux extraits analysés cidessous. Une analyse d'archives et des entretiens avec des acteurs extérieurs au collectif (autres associatifs, acteurs publics, etc.) ont été réalisés mais ne sont pas mobilisés ici, notre parti pris consistant à se concentrer sur les dynamiques que la nécessité de s'entendre et de s'engager sur un argumentaire commun crée à l'intérieur du collectif étudié.

\section{Application à un conflit d'aménagement sur l'étang de Berre}

9 L'implantation de la Société Générale des Huiles et Pétroles à Lavera, en 1922, lance l'essor de la pétrochimie sur le territoire de l'étang de Berre, lequel, sous la planification de l'État français centralisé, va devenir un haut lieu de cette industrie. L'étang de Berre, porté en figure de proue de la modernisation et du développement industriel et technologique par la presse locale et nationale, commence également à être le siège de mobilisations sociales refusant cette vocation exclusivement industrielle. Le 15 janvier 1970, la pêche est interdite dans l'étang, dix jours après l'annonce de l'implantation d'unités sidérurgiques à Fos, ce qui compromet le report éventuel des pêcheurs professionnels vers cette zone limitrophe. Sous l'impulsion de ces derniers, une importante mobilisation éclate (Paillard, 1981), regroupant sous la critique de l'industrialisation de l'étang de la dénonciation des pollutions aériennes, la revendication d'une décentralisation des décisions politiques et la défense des identités régionales. Les premières associations locales de protection de la nature naissent de ce conflit. C'est à la faveur de ce contexte que va se développer, à partir des années 1980-1990, la protestation contre l'usine hydro-électrique de Saint-Chamas (nord de l'étang de Berre).

10 L'étang de Berre est en effet le point de chute, depuis 1966, d'une chaîne hydroélectrique, gérée par Électricité de France (EDF). Cette chaîne prélève l'eau de la Durance, rivière située au nord de l'étang, qu'elle rejette dans celui-ci après turbinage. Ces rejets d'eau douce dans un étang qui communique avec la mer conduisent à la disparition des écosystèmes marins. En 1988, pêcheurs, riverains et associations de protection de la nature se mobilisent pour la résolution de ce problème. Différents plans d'action se succéderont, sans parvenir à une solution durable, les services de l'État considérant tout au long des différentes concertations que le maintien de la 
chaîne représente un intérêt national et que le problème écologique de l'étang ne saurait être résolu par son simple démantèlement. En 2000, le Groupement d'intérêt public pour l'étang de Berre (le GIPREB) est créé en vue d'évaluer les différentes solutions techniques et de mener une concertation pour en retenir une. L'impératif de maintenir la productivité de la chaine hydroélectrique oriente les membres du GIPREB vers une solution de dérivation des rejets hydrauliques vers le Rhône ou la mer. En 2004, le représentant des services de l'Etat au sein du GIPREB propose l'étude d'un canal de dérivation reliant la chaîne hydroélectrique au Rhône, en traversant la plaine de la Crau. Rapidement, le projet s'ébruite et il se dit qu'un canal de quarante mètres de large va traverser les propriétés agricoles. Plusieurs agriculteurs protestent, appuyés par des élus municipaux indignés de n'avoir pas été consultés sur ce projet. De plus, la plaine de la Crau jouxte le territoire des Alpilles dont l'intégrité paysagère et la structure foncière sont le cheval de bataille de plusieurs associations.

11 Certains de ces protestataires connaissent une association de l'étang de Berre qui s'oppose au GIPREB et dénonce dans la presse régionale les projets de dérivation. Cette association est alors invitée à diverses réunions publiques pour présenter la situation à des acteurs oubliés par la communication du GIPREB et fait connaître la solution alternative à la dérivation qu'elle propose. Cette solution prévoit que la chaîne hydroélectrique reste fonctionnelle en ne turbinant qu'un tiers des volumes déversés dans l'étang, les écosystèmes marins pouvant s'adapter à ces volumes. Une dérivation serait dans ce cas inutile. Cette proposition implique de restituer à la Durance le tiers des débits que la chaîne lui prélève, un autre tiers étant restitué aux canaux agricoles de la vallée de la Durance. Cette solution, dite des "trois tiers ", avait été évaluée par le GIPREB deux ans plus tôt et jugée techniquement infaisable et pénalisante pour EDF. Toutefois, un collectif de dix-neuf associations se constitue autour de l'association berroise afin de s'opposer à la dérivation. Au sein de ce collectif, comment l'argumentation joue-t-elle dans l'interaction entre les acteurs issus des premiers conflits et les acteurs émergents sur la scène berroise?

\subsection{L'effet d'engagement des arguments dans l'interdiscursivité du conflit}

12 À sa formation, ce collectif ne définissait pas son action comme une mobilisation pour la défense de l'environnement ni comme la promotion de valeurs proenvironnementales. Progressivement, il a élargi ses activités à la défense des écosystèmes de l'étang de Berre et de la Durance, et finalement entrepris un travail de promotion de l'agro-écologie. Initialement, le seul trait commun à ces acteurs était de refuser la construction d'un canal, alors que les propositions techniques du collectif ne faisaient pas l'objet d'une compréhension partagée par tous les membres. La défense de la proposition des «trois tiers » apparaissait à beaucoup d'entre eux comme une figure obligée de l'espace public (au sens habermassien), une ressource efficace pour faire barrage au canal dans l'espace politique local. Pourtant, les membres du collectif vont progressivement entretenir un nouveau rapport à ces propositions techniques pour finir par les défendre en tant qu'intrinsèquement bonnes pour l'environnement. Alors même que le GIPREB déclare en 2004 que le projet de canal est abandonné, ces acteurs continuent de porter leur revendication car, chemin faisant, ils se sont convaincus que 
la situation environnementale de la Durance et de l'étang de Berre exigent les mesures qu'ils préconisent.

Lorsque le collectif se forme, sa revendication est reçue par les principaux acteurs publics comme symptomatique d'une défense individualiste d'un «chez-soi», sans considération pour l'intérêt public ${ }^{1}$. Pour plusieurs élus locaux et associatifs, ces protestataires portent peu d'intérêt à la réhabilitation de l'étang de Berre et à l'approvisionnement de la région Provence-Alpes-Côte d'Azur en énergie renouvelable. Les membres du collectif s'étaient regroupés autour d'un argumentaire technique qui leur paraissait à la fois une proposition de bon sens et le meilleur moyen d'invalider le projet du canal. Il leur est ainsi rapidement apparu que pour être entendu, cet argumentaire ne suffisait pas, et qu'ils allaient devoir afficher une prise en compte de l'intérêt public. Il ne s'agissait donc pas de réviser leurs propositions, mais de montrer à l'aide de nouveaux arguments que quiconque souhaitant l'équilibre de cette région ne pouvait qu'être favorable à ces propositions.

Pour cela, le collectif va contrer deux objections, celle que des associations majoritairement extérieures à l'étang de Berre n'étaient pas pertinentes ni légitimes pour traiter de la réhabilitation de l'étang et celle de ne pas viser l'intérêt public. Par le passé, l'association berroise initiatrice de la proposition des «trois tiers » défendait celle-ci en avançant qu'en plus de résoudre le problème de l'étang de Berre, elle était bénéfique à l'état écologique de la Durance. De surcroît, en restituant de l'eau limoneuse à la Durance, la solution des trois tiers réduisait l'érosion des plages de Camargue, cette érosion s'expliquant par l'absence de limons transportés par le Rhône, dont la Durance est un affluent. Ces arguments servaient à étayer la défense des propositions techniques en présentant des bénéfices qui iraient au-delà de la résolution du problème de l'étang. En conservant cette ligne d'argumentation, le collectif restait exposé à la critique de son ancrage territorial et aux soupçons d'arguties dissimulant un simple refus de l'ouvrage de dérivation. Mais en déplaçant leur revendication vers la prise en charge de la Durance, avec pour effet supplémentaire de résoudre le problème de l'étang de Berre, l'appartenance géographique des associations du collectif devient pertinente (puisque directement irriguée par l'eau de la Durance). Le canal de dérivation, qui continuerait à priver la Durance de son eau, peut être critiqué sans évoquer ses désagréments pour ses riverains ${ }^{2}$.

C'est incidemment à l'élaboration d'un argumentaire commun que les revendications du groupe vont ainsi évoluer. Alors que le collectif prépare son intervention à une réunion publique, les échanges suivants ont lieu :

- Georges : Sau -sauver la Camargue (.) c'est ça - par où - il faut aller en aval! Sauver la Camargue impose de réhabiliter la Durance pour éviter - pour ne plus avoir de rejet dans l'étang de Berre.

- Benoit : mais, si tu veux -

- Georges : les trois sont liés! Faut pas commencer par l'étang de Berre

- Benoît: non, mais, il faut commencer parce que c'est là que [le GIPREB] nous amène. Avant la Camargue c'est -

- Jean-Laurent : la Durance!

- Georges : oui, mais si tu veux, les gens ce qu'ils vont bien comprendre, si tu veux, les gens disent la Camargue est en danger parce qu'il y a pas de limon. Ça, dans les consciences, ça y est chez les gens. Et les limons, tu les prends où?

- Benoît : dans la Durance

- Georges : voilà !

- Benoît : sauf que, eux, ils prétendent qu'avec leur canal de dérivation - 
- Georges : oui, mais après on a l'argument

- Benoît : oui oui oui ! Georges alors qu'il réfléchit à la meilleure stratégie argumentative. Il reviendra souvent par la suite sur cette solidarité environnementale. En réunion publique, il soutiendra : «Pour sauver l'étang il faut réfléchir au problème de la Camargue qui est étroitement lié au devenir la Durance. Et donc sauver les trois entités, c'est faire confiance tout simplement aux processus naturels ». Cet argument participe à la redéfinition du sens que l'action du collectif prend aux yeux de ses membres. L'idée que l'état de plusieurs territoires relève de manière insoupçonnée d'une même décision d'aménagement, la dérivation de la Durance pour la production d'électricité, fait forte impression sur plusieurs membres. Il apparaît à ceux-ci que restaurer le fonctionnement naturel de la Durance, en arrêtant simplement de dériver son eau dans l'étang de Berre, suffirait à sauver ces trois espaces naturels. Ces membres découvrent ainsi qu'en défendant les solutions du collectif ils ont l'opportunité de faire bien davantage que d'empêcher la construction d'un canal.

Cette idée, qui n'émerge qu'à la faveur de la recherche d'arguments et qu'en réponse à une invocation par leurs opposants de l'intérêt général, contribue à intensifier l'engagement des membres, amenés à se représenter leur action comme intéressant un enjeu plus large ${ }^{3}$. Au fil des argumentations, le collectif va affirmer que le problème de fond est la gestion de la Durance, problème dont l'échelle territoriale est trop vaste pour se satisfaire du GIPREB comme seul interlocuteur. Selon lui, les élus locaux, trop dépendants d'un électorat concerné exclusivement par l'étang de Berre, ne sont ni territorialement pertinents ni aptes à penser l'intérêt général. Le collectif exige donc auprès du Conseil régional la création d'une structure interdépartementale qui s'attèlerait à ce problème. Il répond ainsi aux objections qui lui sont faites et va jusqu'à les renvoyer aux acteurs publics qui en sont à l'origine.

La défense de la rivière introduit également au sein du groupe de nouveaux principes éthiques et moraux pro-environnementaux L'état de la Durance ne s'inscrit que très marginalement dans les missions du GIPREB. Celui-ci peut rejeter l'argumentation du collectif comme hors propos. De plus, la création d'un foyer d'attention publique autour de la Durance est difficile au sein du territoire berrois, déjà en prise à de nombreux enjeux de gestion environnementale. Pour déplacer le débat vers cette thématique, l'état de la Durance doit apparaître comme s'imposant aux acteurs publics, comme une obligation que l'on ne peut plus ignorer, comme un devoir moral que l'on ne choisit pas. C'est la ligne d'argumentation que le collectif va suivre en faisant de la rivière un "patient moral ", prescripteur d'obligations morales, ce qui est un principe central de l'axiologie environnementaliste profonde (deep ecology, Soper 2001). Le fond de l'argumentation du collectif repose en effet sur l'indignation morale devant la dénaturation de la rivière :

il n'y a pas que l'étang de Berre qui souffre de ce qui s'est fait avec l'aménagement, toute la Durance est privée d'eau, c'est lamentable! Elle est dans un état d'insalubrité, d'assèchement, d'appauvrissement, de dégradation. C'est un pipi de chat, la Durance avec $4 \mathrm{~m}^{3} / \mathrm{s}$, c'est rien!

Finalement, le collectif aboutit à un système d'argumentation qui renforce l'engagement de ses membres. Schématiquement, ce système fonctionne à la façon d'une argumentation par engagement ${ }^{4}$ (Walton 2006): qu'un membre refuse la dérivation implique qu'il soutient que la dérivation ne résout pas le problème de la 
Durance, ce qui implique qu'il soutient qu'il faut résoudre le problème de la Durance pour résoudre celui de la Camargue et de l'étang de Berre, ce qui implique qu'il soutient que l'on a collectivement une obligation à sauver ces espaces naturels.

\subsection{Arguments ad hominem et ethos militant dans la controverse technique}

La principale critique adressée au collectif est qu'il ne serait pas possible de restituer son eau à la Durance sans risquer d'accroître les risques d'inondation en cas de forte crue, le lit mineur de la rivière, longtemps asséché, ayant changé de morphologie. Le collectif répond qu'il propose de restituer un débit de $240 \mathrm{~m}^{3} / \mathrm{s}$ alors que les épisodes de fortes pluviométries montrent régulièrement que la rivière peut accroître son débit de $2000 \mathrm{~m}^{3} / \mathrm{s}$ sans inondation. De plus, une étude interministérielle sur la Durance concluait en 2002 que l'absence de crues importantes rendait la rivière d'autant plus dangereuse en cas de forte crue. Selon le collectif, cela signifie que non seulement les restitutions n'augmentent pas les risques d'inondation, mais qu'elles sont nécessaires pour s'en prémunir.

21 En dépit de ces arguments, le collectif ne convainc pas les principaux acteurs publics qui lui opposent que le syndicat mixte d'aménagement de la Vallée de la Durance estime que les restitutions sont néfastes à la rivière et à la sécurité civile. Confrontés à ce qui leur apparaît comme un argument d'autorité et ne comprenant pas les résistances à leurs arguments, les membres du collectif quittent les niveaux cognitif et épistémique de l'argumentation. Cette situation favorise alors l'utilisation d'arguments ad hominem. En effet, le refus d'un élu de reconnaître la possibilité des restitutions à la Durance est réfuté par les caractéristiques personnelles de cet acteur, soit que celui-ci soit accusé de former une alliance politique avec un « défenseur » de la dérivation, soit que son appartenance idéologique le pousse à valoriser, coûte que coûte, les politiques de grands travaux. Tel autre acteur sera accusé de "marcher pour le BTP », d'où son entêtement à nier l'« évidence » des restitutions, lesquelles n'impliquent pas de travaux publics. Les arguments du GIPREB sont rejetés au motif que, la mission de cet organisme consistant à étudier les différentes solutions techniques, une solution « immédiate et évidente », comme celle du collectif, mettrait fin à cette structure. Si un quelconque lien peut être établi entre EDF et un interlocuteur, le collectif réfutera les arguments de ce dernier au motif qu'EDF a intérêt à faire traîner le problème.

Ce mode d'argumentation dispose le collectif à se représenter l'existence d'un conglomérat d'intérêts personnels œuvrant contre la réhabilitation de l'étang. Cela participe à la construction de l'identité collective du groupe comme acteur luttant contre la main-mise des intérêts privés, les militants s'attribuant le rôle d'un « contrepouvoir ».

L'incapacité du collectif à convaincre par des arguments techniques une autre catégorie d'acteurs renforce cet effet des arguments ad hominem. Le collectif est souvent confronté à une objection : comment expliquer que des acteurs dont la probité et la compétence sont indéniables, par exemple des commissaires d'enquête publique, n'adhèrent pas pour autant à ses propositions? Aucun conflit d'intérêt ne peut être évoqué par les membres pour expliquer que les commissaires-enquêteurs ne reconnaissent pas les propositions du collectif comme valides, ce qui rend inopérants les arguments ad hominem traitant ces acteurs d'interlocuteurs «biaisés» (Walton 
2006). Le collectif emploie alors un schéma méta-épistémique expliquant que des interlocuteurs compétents et honnêtes puissent néanmoins leur donner tort. Ces derniers seraient victimes "des mensonges qu'EDF disperse depuis des années " ou utiliseraient des données biaisées produites par l'industriel et diffusées par le GIPREB. Les logiciels de modélisation de courantologie utilisés par les experts seraient euxmêmes faussés, car implémentés en collaboration avec EDF. En somme, des interlocuteurs compétents et honnêtes peuvent donner tort au collectif si les données et les informations qu'ils utilisent sont biaisées à la source. Les militants recourent ainsi à un argument ad hominem d'un type particulier, l'« empoisonnement du puits » (poisoning the well, Walton $1998: 230$ ). Ce mode d'argumentation consiste à rejeter une proposition en raison du locuteur dont elle émane. Pour cela, il vise à établir que ce locuteur est «biaisé » et qu'en conséquence toute proposition émanant de lui doit être tenue pour fragile ou douteuse. Il s'agit alors de réfuter certaines des propositions antérieurement défendues par ce locuteur, celles-ci pouvant n'entretenir aucun lien direct avec la proposition initialement discutée.

Ce mode d'argumentation permet aux militants de rendre intelligible l'insuccès de leurs propositions, tout en désignant l'emprise néfaste d'un industriel, emprise que le collectif sera à même de combattre puisque appartenant au domaine des idées et des discours. Cet argument conforte la revendication des militants d'exercer un contrepouvoir.

Mais l'argument de l'empoisonnement du puits n'est soutenable qu'à condition de présupposer que les locuteurs relayant les arguments et les données biaisés ne sont pas en état de former un jugement réflexif sur leur situation épistémique. Ces locuteurs peuvent soit manquer d'esprit critique, soit être victimes d'une trop forte confiance dans les institutions, soit être aveuglés par une idéologie ou par leur orgueil personnel. Pour le collectif, la capacité d'un acteur à «échapper " aux arguments biaisés, à demeurer vigilant et critique par rapport aux tentatives d'«empoisonnement» du débat, en somme, sa capacité à accéder à la vérité, dépend de ses vertus personnelles.

Les membres du collectif s'attachent aussi bien à dénoncer l'absence de telles vertus chez leurs opposants qu'à en manifester les traits dans leur propre comportement car eux-mêmes ne peuvent jamais être sûrs de ne pas être des interlocuteurs " honnêtement » biaisés. Pour cela, la pratique militante est mise en correspondance avec les critères du jugement. Qu'un opposant au collectif revienne sur son évaluation des projets de dérivation ou qu'il mentionne les propositions du collectif dans son argumentaire, le collectif y verra un "retournement de vestes ", une tentative de ne pas "perdre la face». Inversement, pour les membres du collectif, accueillir avec incrédulité les explications du GIPREB, montrer que l'on «ne s'en laisse pas conter ", c'est faire montre d'un esprit critique qui préserve le jugement de l'emprise de données et d'arguments biaisés. La fidélité à un engagement collectif se joint à la fermeté du jugement pour faire de l'invariabilité des points de vue une vertu épistémique militante. Le choix d'autres associatifs de limiter leur action à la participation aux instances de concertation, sans les remettre en cause, est vu par les membres du collectif comme la preuve d'un manque de tempérament ou d'une servilité coupable qui a pour conséquence une faiblesse du jugement. Aussi les membres du collectif prennent-ils soin de cultiver leur attitude critique envers les institutions et sanctionnent toute attitude contraire au sein du groupe en accusant de "grande naïveté » celui qui serait conciliant envers un acteur public. 
27 Les militants tiennent la capacité à avoir une vue globale des problèmes pour une vertu préservant des raisonnements biaisés. Les détracteurs du groupe ne verraient pas, selon celui-ci, les multiples facettes du problème, telles que l'effet de la libéralisation du marché énergétique sur la production d'EDF. Les acteurs (associatifs) qui exercent une expertise technique et qui néanmoins se fient aux données produites par le GIPREB ou par EDF sont accusés de ne pas voir ce qui se joue réellement. À l'inverse, les membres du collectif développent un pluri-militantisme ou convoquent dans leurs arguments des thématiques débordant le cadre de la résolution technique du problème, par exemple la privatisation des régies de traitement de l'eau en Europe. Cette vigilance à l'élargissement des causes entrant en ligne de compte est valorisée comme une qualité propre du militant ou plus généralement du citoyen vigilant préservant de la naïveté et prémunissant des analyses trop rapides soufflées par les acteurs publics aux populations locales. Elle pourrait également se lire comme un travail sur la portée des arguments (Chateauraynaud 2011); dans le cadre qui nous occupe, on notera seulement que cette argumentation appuie la construction d'un ethos militant dans le groupe.

Le militantisme peut se comprendre comme une inscription dans le débat politique de problématiques jusque lors traitées techniquement par des instances localisées. Il convient donc d'étudier comment l'apport de cet ethos à l'identité collective du groupe se double d'une convergence des militants sur le sens politique qu'ils donnent à leur action et sur les actions qu'ils entendent mener ensemble. Or d'importants travaux ont montré que les conversations entre membres d'un groupe protestataire (spécialement les conversations de sociabilité) sont des formes discursives essentielles pour l'inscription de l'action collective dans la durée (Cefaï 2007, Hunt et Benford 1994, Jaspers 1997, Lichterman 1999). De plus, Chateauraynaud (2011) montre que l'argumentation peut opérer un rapprochement entre acteurs à mesure que ceux-ci interagissent à l'intérieur de différents cadres discursifs, la conversation fournissant un de ces cadres. Nous reprenons ci-après ces deux questionnements en restant exclusivement au niveau des conversations internes à un groupe. Nous étudions la possibilité que les échanges argumentatifs, tout en prenant une forme discursive différente de celle d'un débat contradictoire ou d'un débat public, contribuent à la convergence et à la sociabilité entre les militants du collectif observé.

\subsection{Convergence et sociabilité dans l'argumentation entre militants}

Les échanges argumentatifs occupent une place importante dans l'activité interne du collectif. Les militants argumentent parfois à propos de propositions sur lesquelles ils savent partager le même avis. Le déroulement de ces échanges nous est apparu suivre les mêmes principes que les conversations familières (Traverso 1996). Un thème est introduit par un locuteur. Il est ensuite amplifié par des glissements thématiques établis successivement sur la base d'un segment de l'énoncé précédent. Ces glissements thématiques conduisent à l'introduction d'un nouveau thème permettant à la conversation de ne pas s'épuiser. Dans les conversations de sociabilité, notamment les échanges à bâtons rompus, les participants observent une « contrainte d'enchaînement maximal " (ibid.): les glissements thématiques doivent maintenir le plus longtemps possible la conversation et permettre la participation la plus large possible.

Dans le collectif observé, de nombreux échanges prennent cette forme, mais les glissements entre énoncés se font à travers une relation d'étayage : chaque membre 
emboîte le pas de l'interlocuteur précédent en confirmant ou en complétant son argumentaire. L'introduction du thème ouvrant l'échange se fait le plus souvent en rapportant les propos d'un opposant. Parfois, le président du collectif lance l'échange en affirmant une proposition assez lourde de sens mais en ne livrant aucun élément à l'appui de celle-ci, ce qui, inévitablement, appelle les autres membres à procéder à une relance. L'échange s'amplifie alors à mesure que le président livre un à un les éléments qui étayent son premier énoncé, éléments tour à tour complétés et confirmés par les autres membres.

On peut observer cette argumentation entre militants convaincus dans les échanges suivants qui naissent du commentaire qu'un membre fait d'une coupure de presse où s'exprime un représentant du MNLE (Mouvement national de Lutte pour l'Environnement). Le MNLE militait alors pour un siphon déversant en mer les rejets de la chaîne hydroélectrique. Le MNLE est tenu par le collectif pour favorable aux intérêts d'EDF.

[Ouverture de l'échange avec proposition de deux thèmes : le MNLE et le Siphon] ${ }^{5}$

1. Robert : qu'est-ce qu'il porte comme message lui, le siphon?

2. Benoît : il veut la dérivation ou un siphon

3. Jean-Laurent : c'est le MNLE !

4. Vernon : c'est le GIPREB ! c'est un giprebiste

[introduction du thème "siphon"]

5. Robert : le siphon

[ratification du thème siphon]

6. Jean-Laurent : le siphon!

[amplification thématique par étayages successifs]

7. Benoît : non, mais attend le siphon, c'est vraiment siphonné !

8. Robert : non, mais c'est bien, c'est une bonne idée, il faut 15 ans pour le faire !

9. Benoît : non, mais attend c'est infaisable un siphon!

10. Vernon: non, mais là toute façon les limons, une fois que les limons sont dans les -

11. Robert [ironique] : tout est faisable

12. Benoît : non!

13. Robert : si c'est < se fait couper la parole >

14. Benoît: non! non! moi je te garantis que c'est pas possible de faire un siphon. C'est pas possible! D'abord, tu sais pas ce que tu vas rencontrer dessous et tu sais pas comment tu vas sortir. Et si tu sors vraiment en mer alors là attention! c'est plus que le tunnel sous la Manche! [l'énoncé "faut être fou (...)" permet de glisser au second thème : le MNLE]

Non, mais attends! il faut être fou pour proposer ça! Fou ou alors il faut vouloir qu'on ne touche pas à EDF

[introduction du theme : le MNLE]

15. Robert: tu sais je vais te dire j'ai discuté moi avec le MNLE, ils sont pour l'incinération [des déchets] aussi !

[ratification du thème ]

16. Jean-Laurent : oui oui tout à fait !

[amplification du thème]

18. Jean-Laurent: on l'a vu quand on y est allé pour la journée SPPPI. Le MNLE il disait « oh c'est des - rejets, c'est pas énorme, y a pas de grosses » < se fait couper la parole >

19. Robert: «y a pas de preuve que les incinérateurs polluent ». Y a aucune preuve solide hein

La conviction partagée des membres quant à l'irréalisme du siphon ne fait pas de doute. Le collectif existe alors depuis plusieurs années et chaque membre sait que les autres membres rejettent cette proposition. Pourtant les militants formulent des arguments, 
que tous connaissent déjà, pour rejeter cette "solution ». La manœuvre de Benoît au tour de parole 14 correspond à un procédé d'amplification de l'échange : il feint de ne pas comprendre l'intention ironique de Robert pour détailler son argumentaire.

Que rend possible dans le collectif ce type d'échanges? En premier lieu, on observe généralement que lors de ces échanges le cadre d'interaction s'assouplit. Des apartés entre acteurs ont lieu, certains membres introduisent des anecdotes personnelles au milieu de conversations portant sur la faisabilité technique d'une dérivation. D'autres, habituellement silencieux lors de l'élaboration de tracts ou de dossiers d'information, prennent la parole. La prosodie change également vers davantage de cordialité. Il paraît raisonnable de considérer que ces échanges, tout en conservant une forme argumentée, participent à la sociabilité entre les militants. En prenant pour support l'activité principale du groupe, c'est-à-dire la défense de leurs propositions, cette sociabilité reste ainsi inscrite dans le champ de l'action collective. Plusieurs membres ne se fréquentent que dans le cadre de ces moments d'échanges. Peut-être ne sauraient-ils par quelle activité prolonger le plaisir de ce lien s'ils se rencontraient en dehors du cadre du collectif. Un rapport circulaire existe entre la sociabilité et l'action collective : la première naît de la seconde et a besoin de sa poursuite pour continuer à s'exercer, en retour la sociabilité donne l'intensité nécessaire à l'action collective pour maintenir l'engagement collectif lorsque la situation stagne ou que les militants rencontrent des difficultés. Ici, l'argumentation fournit un support à ce processus de renforcement de l'action collective.

L'argumentation entre militants déjà convaincus remplit un autre rôle: doter les militants d'un mode d'interaction qui leur est propre, d'une routine interactionnelle qui leur permet d'interagir en tant que membres. Par ces étayages successifs, c'est également l'« esprit » du groupe qui est affirmé, le sentiment fort et continuellement confirmé que ces acteurs forment un groupe pourvu d'une conception commune et d'une intention partagée. Un membre, incapable de contribuer à la rédaction de tracts ou à toute autre activité militante, pourra néanmoins participer à ce type d'échanges et ainsi donner corps à son engagement.

Les échanges argumentatifs entre militants déjà persuadés ne sont-ils pour autant qu'un support relationnel de l'engagement collectif, sans effet sur le contenu de l'action collective ? Ces échanges, même s'ils portent sur des contenus déjà partagés par les militants, participent néanmoins à la définition du sens que les acteurs donnent à leur action collective. L'échange retranscrit plus haut se poursuit sur le thème du MNLE. Cette association étant connue pour ses liens avec la CGT et le PCF, l'échange glisse sur la conception écologique du parti communiste, accusé de privilégier le maintien de l'outil industriel, source d'emplois, sur la préservation de l'environnement. De là, la conversation passe aux dérives de la vision productiviste de l'économie. Un militant appuie les dires d'un autre avec l'exemple de l'administration de Ceausescu, ce qui autorise un glissement sur l'autoritarisme et la liberté de parole. Les militants ne prennent pas au pied de la lettre ces propos qui sont de l'ordre de la conversation à bâtons rompus. Aucun d'eux ne songe à rapprocher le conflit d'aménagement de l'étang de Berre de la situation de la Roumanie d'avant 1989. Toutefois, ces échanges offrent aux militants l'occasion de converger sur une dénonciation du productivisme et de verbaliser leur refus de la place occupée par les services de l'État et les élus locaux dans l'aménagement du territoire, toutes proportions gardées. Dans de tels échanges, les militants constatent qu'ils partagent certaines idées et valeurs qui dépassent le cadre 
de leur mobilisation initiale. Ils peuvent souhaiter entamer des actions motivées par ces valeurs, ce que certains d'entre eux ont d'ailleurs fait, avec un comité de soutien à José Bové et une liste commune aux élections législatives.

\section{Conclusion}

L'étude de cas que nous avons présentée suggère que l'argumentation peut contribuer à la formation durable de groupes militants à partir de simples protestations ponctuelles et hétérogènes. Le rôle ainsi joué par l'argumentation ne se comprend pas dans les termes d'une causalité mécanique. Il convient plutôt d'y voir la résultante de l'investissement par des acteurs de la pluralité des dimensions des échanges argumentatifs. Ces échanges constituent tout à la fois une procédure collective de raisonnement, un mode de relation sociale entre personnes engagées à agir ensemble et une mise en relation les valeurs et les rationalités d'espaces sociaux conflictuels, y compris lorsque ceux-ci ne communiquent pas entre eux. Nous nous sommes intéressés à la valeur cohésive des échanges argumentatifs entre militants d'un même groupe protestataires et la capacité de ces échanges à permettre, quasi incidemment, une évolution de l'intention collective de ce groupe et des principes qu'il défend.

Ce n'est toutefois qu'un aspect de l'effectivité sociale de l'argumentation. D'autres approches auraient pu analyser ce cas d'étude à partir de procédures de cadrage ou de mise en intrigue dans la constitution d'un problème public, en insistant davantage sur le jeu d'acteurs et le foyer de tensions environnementales que constitue l'étang de Berre. Une approche transversale aux différents espaces-temps de ces conflits aurait également pu être menée. Dans ce cas, inversement, les processus intra-groupaux seraient placés au second plan, le matériau langagier analysé différant à son tour. Par exemple, l'analyse de l'amplification thématique dans les échanges à bâtons rompus et de sa contribution à l'élargissement des revendications du groupe nous parait impliquer de se concentrer sur une échelle empirique microsociologique et synchronique. Pour autant, il s'agit davantage de choix imposés par les limites concrètes d'une recherche limitée dans le temps. Du point de vue d'un programme argumentatif en sociologie, ces différentes approches ne nous paraissent aucunement incompatibles, que ce soit sur le plan théorique ou sur le plan empirique, voire, se nécessitent mutuellement.

\section{BIBLIOGRAPHY}

Amossy, Ruth. 2006. L'argumentation dans le discours (Paris : Colin)

Boltanski, Luc \& Laurent Thévenot. 1991. De la justification. Les économies de la grandeur (Paris : Gallimard)

Bouvier, Alban. 1997. « Argumentation et cognition en sociologie morale et juridique », Raymond Boudon, Alban Bouvier \& François Chazel (éds), Cognition et sciences sociales (Paris : PUF), 91-119 
Bouvier, Alban. 1999. Philosophie des sciences sociales. Un point de vue argumentativiste en sciences sociales(Paris : PUF)

Cans, Roger. 2006. Petite histoire du mouvement écolo en France (Paris : Delachaux-Niestlé)

Cefaï, Daniel \& Claudette Lafaye. 2002. « Les répertoires d'argumentation et de motivation dans l'action collective. Le cadrage d'un conflit urbain à Paris », Daniel Cefaï \& Isaac Joseph (éds). L'héritage du pragmatisme (La Tour-d'Aigues : Éd. de l'Aube), 371-394

Cefaï, Daniel. 2007. Pourquoi se mobilise-t-on? Les théories de l'action collective (Paris : La Découverte)

Chateauraynaud, Francis. 2007. « La contrainte argumentative. Les formes de l'argumentation entre cadres délibératifs et puissances d'expression politiques ", Revue européenne des sciences sociales $136,129-148$

Chateauraynaud, Francis. 2011. Argumenter dans un champ de forces. Essai de balistique sociologique (Paris : Pétra)

Conein, Bernard. 2005. Les sens sociaux (Paris : Economica)

Dewey, John. 1993. Logique. La théorie de l'enquête. (Paris : PUF)

Doury, Marianne. 2012. "Preaching to the Converted. Why Argue When Everyone Agrees?", Argumentation $26: 1,99-114$

Groethuysen, Bernard. 1977 [1927]. Origines de l'esprit bourgeois en France (Paris : Gallimard)

Hunt, Scott \& Robert D. Benford. 1994. « Identity Talk in the Peace and Justice Movement », Journal of Contemporary Ethnography 22 : 4, 488-517

Jasper, James. 1997. The Art of Moral Protest : Culture, Biography, and Creativity in Social Movements (Chicago : University of Chicago Press)

Lafaye, Claudette \& Laurent Thévenot. 1993. « Une justification écologique ? Conflits dans l'aménagement de la nature ", Revue française de sociologie 34 : 4, 495-524

Lichterman, Paul. 1999. « Talking Identity in the Public Sphere: Broad Visions and Small Spaces in Identity Politics », Theory and Society $28: 1,101-141$

Orkibi, Eithan. 2008. «Ethos collectif et Rhétorique de polarisation : le discours des étudiants en France pendant la guerre d'Algérie ", Argumentation et Analyse du Discours 1 [en ligne : http:// aad.revues.org/438]

Paillard, Bernard. 1981. La damnation de Fos (Paris : Seuil)

Rennes, Juliette. 2011. « Les formes de la contestation. Sociologie des mobilisations et théories de l'argumentation ", A contrario 16, 151-17

Simon, Gilles. 2010. Plogoff. L'apprentissage de la mobilisation sociale (Rennes : Presses Universitaires de Rennes)

Soper, Kate. 2001. «Écologie, nature et responsabilité », La Revue du M.A.U.S.S. 17, 71-93

Stewart, Charles J., Craig A. Smith \& Robert E. Denton. 2007. Persuasion and Social Movements (Long Grove, IL : Waveland Press)

Traverso, Véronique. 1996. La conversation familière. Analyse pragmatique des interactions (Lyon : Presses Universitaires de Lyon)

Trom, Danny. 1999. « De la réfutation de l'effet NIMBY considérée comme une pratique militante. Notes pour une approche pragmatique de l'activité militante », Revue française de science politique $49: 1,31-50$ 
Walton, Douglas. 1998. Ad Hominem Arguments (Tuscaloosa, AL : University of Alabama Press)

Walton, Douglas. 2006. Fundamentals of Critical Argumentation (Cambridge : Cambridge University Press)

Weber, Max. 1963 [1919]. Le savant et le politique (Paris : 10/18)

Zagzebski, Linda. 2005. « Les vertus épistémiques », Julien Dutant \& Pascal Engel (éds), Philosophie de la connaissance. Croyance, connaissance, justification (Paris : Vrin), 395-419

\section{NOTES}

1. Sur l'usage rhétorique de l'accusation de NIMBY, cf. Trom (1999).

2. On peut voir en cela un procédé de montée en généralité (Boltanski et Thévenot 1991).

3. Suivant une logique d'enquête collective (Dewey 1993), la production de connaissances par le groupe peut s'étalonner sur les réfutations, doutes ou confirmations qu'il rencontre au cours de son action.

4. Cette argumentation consistant à faire reconnaître par un interlocuteur que la proposition qui est soumise à son acceptation se déduit de propositions qu'il a antérieurement soutenues ou que celles-ci se déduisent de cette première, et que, de ce fait, il doit adopter le point de vue qui lui est soumis.

5. Les annotations entre crochets et en italique repèrent les opérations d'amplification et de glissement thématiques ainsi que l'introduction de nouveaux thèmes.

\section{ABSTRACTS}

This article discusses the role of argumentation in the development of environmental activism during land-development conflicts. Argumentation is analyzed here on three levels: First, protest groups must integrate and incorporate pro-environmental content within their own arguments in order to refute their opponents' claims. Second, the controversy leads activists to use different forms of ad hominem arguments, which consolidate the activists' own ethos. Last, protesters use argumentation as a form of sociability. By way of confronting ideas and opinions in a sociable mode, the collective process of reasoning enforces micro-sociological dynamics, and leads to group solidification.

Cet article aborde le rôle de l'argumentation dans le développement du militantisme environnementaliste à l'occasion de conflits d'aménagement. L'argumentation peut jouer à trois niveaux. En premier lieu, les groupes protestataires sont amenés à intégrer à leur argumentaire des contenus pro-environnementaux afin de répondre à leurs contradicteurs. En second lieu, la controverse favorise le recours à différentes formes d'arguments ad hominem qui confortent l'affirmation d'un éthos militant. Enfin, les militants investissent l'argumentation comme une forme de sociabilité. Par la confrontation des idées et des opinions sur un mode sociable, le processus collectif de raisonnement sous-tend les dynamiques micro-sociologiques et renforce la cohésion du groupe. 
INDEX

Keywords: activism, ad-hominem, argumentation, collective ethos, environmentalism, interdiscursivity, land-use conflicts

Mots-clés: ad-hominem, argumentation, conflits d'aménagement, environnementalisme, ethos collectif, interdiscursivité, militantisme

\section{AUTHOR}

\section{AURÉLIEN ALLOUCHE}

Université Aix-Marseille, Laboratoire méditerranéen de Sociologie (LAMES), UMR 7305 